IHBSS/IBBS and STI/HIV vulnerability (especially for trans men), wherein trans people are part of the research team, to provide disaggregated data for trans women/men. A study on the possible drug interaction of anti-retroviral therapy and hormone replacement therapy, and a baseline study on the state of mental health among trans people through participatory action research should be done. Lastly, there should more trans-driven and trans-focused researches, and research grant opportunities.

Conclusion Addressing trans issues should be multi-faceted approach with focus on trans health research/surveillance. Therefore, this regional advocacy agenda can be used by trans groups, program implementers, researchers, and key stakeholders in developing prioritised trans-specific advocacies on research/surveillance.

\section{P6.03 A REGIONAL ADVOCACY AGENDA ON TRANSGENDER HEALTH RESEARCH AND SURVEILLANCE AMONG TRANSGENDER PEOPLE IN THE ISEAN REGION}

RN Cortes. The Philippine Ngo Council on Population, Health and Welfare, Inc., Pasay City, Philippines

\subsection{6/sextrans-2017-053264.655}

Introduction The ISEAN-Hivos Program (IHP) is a regional Global Fund AIDS grant focused on community systems strengthening (CSS) among males having sex with males (MSM) and transgender (TG) organisations in Indonesia, Malaysia, Philippines and Timor Leste. One of the strategies of the program is to disaggregate transgender people from MSM, and be recognised as a unique key affected population. Methods Given the lack of evidence-based data to guide development partners, government agencies and donors to develop trans-specific health interventions, IHP through the ISEAN Secretariat conducted the TransISEAN: Regional Community Workshop on Health, SOGIE and Rights among Transgender People in the ISEAN Region last August 14-16, 2015 in Kuala Lumpur, Malaysia. The objective of the regional consultation is to develop a regional advocacy agenda for trans people that is prioritised on the key areas of trans health and services, and trans research and surveillance.

Results The prioritised advocacy agenda on trans health and services are the following: Sensitisation of healthcare workers and facilities for trans people to ensure confidentiality and recognition of their identities. Development of trans-health and SOGIE IEC materials and strengthening the peer education program for trans youth. Development of a blueprint for the establishment of a Transgender Wellness Centre and transPLHIV support system; and developing a Service Delivery Network for other trans services including psychosocial, gender reaffirming procedures, legal concerns, etc. There should be continuous capacity building of trans groups for trans-led health service delivery. Lastly, SOGIE should be mainstreamed, localised and integrated in the health education and curriculum.

Conclusion Addressing trans issues should be multi-faceted approach with focus on transgender health, including sexual health services. Therefore, this regional advocacy agenda can be used by trans groups, program implementers, researchers, and key stakeholders in developing prioritised trans-specific health advocacies.
P6.04 ABSTRACT WITHDRAWN

\section{P6.05 A SEXUAL REVOLUTION IN PARADISE ? INDIGENOUS YOUTH AND THE DIGITAL AGE!}

${ }^{1}$ Walker D, ${ }^{1}$ Pereznieto P, ${ }^{1}$ Bergh G, ${ }^{2}$ Smith K, ${ }^{3}$ Les F. ' $O$ verseas Development Institute, London, UK; ${ }^{2}$ Plan International, APIA - Western Samoa; ${ }^{3}$ Asia Pacific Indigenous Youth Network, Baguio, Philippines

\subsection{6/sextrans-2017-053264.656}

Introduction Sexual health organisations are embracing social media on an unprecedented scale to engage communities in a more interactive style and with the aim of improving outcomes. However, such technology is still in its early stages and evidence of its efficiency is limited. Especially for young people and underserved communities such as indigenous peoples. The study is an overview of the peer-reviewed evidence on social media to inform consumers in sexual health with a particular focus on the Pacific youth context. Research questions: (1) What is the evidence of benefit for social media campaigns used in sexual health promotion? and (2) What social media campaigns have been used in Indigenous-focused sexual health promotion in the Pacific and what is the evidence of their effectiveness and benefit?

Methods We conducted a scoping study of peer-reviewed evidence. We examined the available literature, conducted researcher surveys, and debriefs, case studies and interviews. This was further accompanied by a consultation of stakeholders. Data collection was still underway in 2016.

Results The review identified 17 intervention studies and seven systematic reviews that met criteria, which showed limited evidence of benefit. We found five projects with significant social media coverage targeting the Indigenous Pacific population for sexual health promotion purposes meeting criteria. No evidence of benefit was found for these projects.

Conclusion Although social media technologies have the unique capacity to reach young people, indigenous communities, and other underserved populations, evidence of their capacity to do so is limited. Current initiatives are neither evidence-based nor widely adopted. Sexual health organisations should tailor platforms specifically to indigenous youth to ensure cultural competencies are met and encouraged.

\section{P6.06 VULNERABILITY TO STI/HIV/AIDS IN ADOLESCENTS IN POVERTY SITUATION}

Izaildo Tavares Luna, Maria Isabelly Fernandes da Costa, Raelson Ribeiro Rodrigues, Patrícia Neyva da Costa Pinheiro. Universidade Federal do Ceará, Fortaleza - CE, Brazil

\subsection{6/sextrans-2017-053264.657}

Introduction This survey has as objective to identify if the adolescents in poverty situation are vulnerable to STI/HIV/ AIDS.

Methods The survey was descriptive, exploratory, with a quantitative approach, carried out with 287 adolescents in a public school situated in a region of low human development rate in Fortaleza-CE, Brazil. The data collection was done through two questionnaires, one sociodemographic and one about the adolescents' vulnerability. It was attributes ponctuations from 0 to 2 points, which, in the end, can present points from 0 (low vulnerability) and 21 (high vulnerability). The data were 